PROCEEDINGS OF THE

AMERICAN MATHEMATICAL SOCIETY

Volume 132, Number 8 , Pages $2433-2439$

S 0002-9939(04)07432-5

Article electronically published on March 4, 2004

\title{
UNIQUENESS OF TRAVELLING WAVES FOR NONLOCAL MONOSTABLE EQUATIONS
}

\author{
JACK CARR AND ADAM CHMAJ \\ (Communicated by Mark J. Ablowitz)
}

\begin{abstract}
We consider a nonlocal analogue of the Fisher-KPP equation $u_{t}=J * u-u+f(u), x \in R, f(0)=f(1)=0, f>0$ on $(0,1)$,

and its discrete counterpart $\dot{u}_{n}=(J * u)_{n}-u_{n}+f\left(u_{n}\right), n \in Z$, and show that travelling wave solutions of these equations that are bounded between 0 and 1 are unique up to translation. Our proof requires finding exact a priori asymptotics of a travelling wave. This we accomplish with the help of Ikehara's Theorem (which is a Tauberian theorem for Laplace transforms).
\end{abstract}

\section{INTRODUCTION}

In this paper we study uniqueness of travelling waves of two nonlocal models. One is the integrodifferential equation

$$
u_{t}=J * u-u+f(u),
$$

where $J * u(z)=\int_{R} J(z-y) u(y) d y$. About $f$ we assume that it is monostable, i.e., $f(0)=f(1)=0$ and $f>0$ on $(0,1)$, e.g., $f(u)=u-u^{2}$, and also that $f^{\prime}(r) \leq f^{\prime}(0)$ for $r \in(0,1)$. We assume that $J \geq 0$ is even, compactly supported and $\int_{R} J=1$. The second model is a discrete version of (1.1), namely, an infinite ODE system

$$
\dot{u}_{n}=(J * u)_{n}-u_{n}+f\left(u_{n}\right), n \in Z,
$$

where $(J * u)_{n}=\sum_{|i| \geq 1} J(n-i) u_{i}$ and $J$ and $f$ are as before. These equations are two versions of the well-studied Fisher-KPP equation ([9], [10])

$$
u_{t}=u_{x x}+f(u)
$$

see, e.g., 3] and the references therein. All the above equations model, e.g., population dynamics (see [8] for a derivation of (1.1)). In 8], (1.3) is actually derived from (1.1): one can formally expand $J * u-u$ into power series, namely $\int_{R} J(y)(u(z+y)-u(z)) d y=c_{1} u^{\prime \prime}(z)+c_{2} u^{\prime \prime \prime \prime}(z)+\ldots$, where $c_{1}=\frac{1}{2} \int_{R} y^{2} J(y) d y$, $c_{2}=\frac{1}{24} \int_{R} y^{4} J(y) d y$, etc., and consider just the first term in the expansion.

We consider solutions which are travelling waves of the form $u(z-c t), z \in R$ for (1.1) and $u(n-c t), n \in Z$ for (1.2), and which connect the steady states 0 and 1 .

Received by the editors August 6, 2002 and, in revised form, May 7, 2003.

2000 Mathematics Subject Classification. Primary 92D15, 39B99, 45G10.

This work was supported by a Marie Curie Fellowship of the European Community IHP programme under contract number HPMFCT-2000-00465 and in part by NSF grant DMS-0096182. 
Let $x=z-c t$ for (1.1) and $x=n-c t$ for (1.2). Then $u(x)$ satisfies

$$
-c u^{\prime}=J * u-u+f(u), u(-\infty)=1, u(+\infty)=0,
$$

where for (1.2) $J * u(x)$ can be formally interpreted as

$$
\int_{R} \sum_{|i| \geq 1} J(i) \delta(x-y+i) u(y) d y .
$$

The existence of solutions of (1.4) has been addressed in some previous papers.

Proposition 1.1. Assume that $J$ has compact support and $f^{\prime}(r) \leq f^{\prime}(0)$ for $r \in(0,1)$. In addition, for (1.2) we assume that $\operatorname{supp} J$ contains either 1 or two relatively prime integers. Then a solution $u$ of (1.4) such that $u^{\prime}<0$ exists for every $c \geq c_{0}$, where

$$
c_{0} \equiv \min \left\{\lambda>0: \frac{1}{\lambda}\left[\int_{R} J(y) e^{\lambda y} d y+f^{\prime}(0)-1\right]\right\} .
$$

Proof. In 11, this is proved for (1.1) and $c>c_{0}$ using the monotone iteration method. One can then obtain the existence of a solution for $c=c_{0}$ using a limiting argument, as is done in, e.g., 4]. In 15, the authors construct travelling wave solutions for (1.2) with $\operatorname{supp} J=\{-1,1\}$ and $c \geq c_{0}$ using a degree argument. For (1.2) with $J$ such that supp $J$ contains 1 or two relatively prime integers, one can first use either of the above techniques to first obtain a solution such that $u^{\prime} \leq 0$, and then add a comparison argument as in [2, p. 290, to show that actually $u^{\prime}<0$.

Similar methods were used to establish existence of travelling waves of some other nonlocal models with monostable dynamics; see, e.g., [1, 4], 6], [13, 11], 14, 5.

In this paper we focus only on (1.1), which is probably the most direct extension of the Fisher-KPP equation. We feel that stating our results for a more general class of monostable nonlocal equations, as in, e.g., 11], would only make the paper unnecessarily harder to read. Therefore, the adaptation of these results to other nonlocal models of monostable type is left to the reader.

The first uniqueness result, for a particular monostable nonlocal model, appeared in the work of Diekmann and Kaper [6]. The authors proved that travelling waves with noncritical speeds (i.e., $c>c_{0}$ ) are unique in the class of monotone solutions. Recently, Xinfu Chen and J.-S. Guo [5] obtained a complete uniqueness result for travelling waves for a generalized discrete version of the nonlocal monostable equation. To be more precise, they show that all such solutions, including nonmonotone waves and the one with the critical speed $c_{0}$, that are bounded between 0 and 1 are unique up to translation.

In this work we extend the method of Diekmann and Kaper to obtain a similar, complete result such as the one in [5]. Let us discuss the differences between the two approaches. The "strategy" of the two methods is similar: first one establishes an a priori asymptotic behaviour of a solution at 0 , then two possible solutions are appropriately compared and shown to be the same. In [5], the authors first show that a solution $u$ satisfies $u^{\prime}<0$ and $\lim _{x \rightarrow \infty} \frac{u^{\prime}(x)}{u(x)}=-\alpha(c)$ (see below for the definition of $\alpha(c)$ ). Then, assuming $u_{1}$ and $u_{2}$ are two solutions, they study $\frac{u_{1}(x)}{u_{2}(x)}$ using a moving plane argument to conclude that $u_{1} \equiv u_{2}$. 
In our work, we first obtain the exact asymptotic behaviour of a solution. We show that the convergence to 0 of any travelling wave is $O\left(e^{-\alpha(c) x}\right)$ for $c>c_{0}$ and $O\left(x e^{-\alpha\left(c_{0}\right) x}\right)$ for the critical speed, as $x \rightarrow \infty$. Note that this is stronger than $\lim _{x \rightarrow \infty} \frac{u^{\prime}(x)}{u(x)}=-\alpha(c)$. With this information at hand, assuming $u_{1}$ and $u_{2}$ are two solutions of (1.4), it then suffices to show that the quotient of $u_{1}-u_{2}$ over the asymptotic rate of convergence to 0 of $u_{1}$ and $u_{2}$ cannot have a maximal point at which it is positive; therefore $u_{1} \equiv u_{2}$. Both parts of our method are shorter than the corresponding ones in [5]. The main and crucial difference is that to obtain the exact asymptotics we construct a Laplace transform representation of a solution and then use the powerful Tauberian Ikehara's Theorem.

\section{UNIQUENESS}

Theorem 2.1. Assume that $J$ and $f$ are as in Proposition 1.1. Then a solution $u$ of (1.4) such that $0 \leq u \leq 1$ exists only for $c \geq c_{0}$ and is unique up to translation.

Proof. First, by a standard comparison argument, one can show that any solution $u$ such that $0 \leq u \leq 1$ actually satisfies $0<u<1$.

Note that the function $g(\lambda) \equiv \frac{1}{\lambda}\left[\int_{R} J(y) e^{\lambda y} d y+f^{\prime}(0)-1\right]$ is positive for all $\lambda>0$. Also, if $g^{\prime}\left(\lambda_{0}\right)=0$ for some $\lambda_{0}$, then

$$
g^{\prime \prime}\left(\lambda_{0}\right)=\frac{1}{\lambda_{0}} \int_{R} y^{2} J(y) e^{\lambda_{0} y} d y>0 .
$$

Thus for every $c>c_{0}, c \lambda+\int_{R} J(y) e^{\lambda y} d y+f^{\prime}(0)-1$ has two negative roots: $-\beta(c)<-\alpha(c)<0$, and a double root $\alpha\left(c_{0}\right)$ for $c=c_{0}$. We first show that any solution of (1.4) such that $0 \leq u(x) \leq 1$ satisfies, after translation,

$$
\lim _{x \rightarrow \infty} \frac{u(x)}{x e^{-\alpha\left(c_{0}\right) x}}=1 \text { and } \lim _{x \rightarrow \infty} \frac{u(x)}{e^{-\alpha(c) x}}=1 \text { for } c>c_{0} .
$$

We begin with the following lemma.

Lemma 2.2. Any solution $u$ of (1.4) with $0 \leq u(x) \leq 1$ is such that $u(x)=$ $O\left(e^{-\gamma x}\right)$ as $x \rightarrow \infty$, for some $\gamma>0$.

Proof. The idea of the proof is to first show that $v(x) \equiv \int_{x}^{\infty} u(s) d s$ is such that $v(x)=O\left(e^{-\gamma x}\right)$ as $x \rightarrow \infty$, for some $\gamma>0$, and then that this also implies that $u(x)=O\left(e^{-\gamma x}\right)$ as $x \rightarrow \infty$.

We first show that $u$ is integrable on $(x, \infty)$. By integrating (1.4) from $x$ to $y$ we get

$$
c(u(x)-u(y)) \geq \int_{x}^{y}[J * u(s)-u(s)] d s+a \int_{x}^{y} u(s) d s
$$

for some $a>0$ depending on $x$ (since $f(u) \geq a u$ ). Note that

$$
\begin{aligned}
& \int_{x}^{y} \int_{R} J(z)(u(z+s)-u(s)) d z d s=\int_{x}^{y} \int_{R} J(z) \int_{0}^{1} z u^{\prime}(s+t z) d t d z d s \\
= & \int_{R} z J(z) \int_{0}^{1}[u(y+t z)-u(x+t z)] d t d z \rightarrow-\int_{R} z J(z) \int_{0}^{1} u(x+t z) d t d z
\end{aligned}
$$

as $y \rightarrow \infty$ by Fubini's Theorem and Lebesgue's Dominated Convergence Theorem. Thus $u$ is integrable on $(x, \infty)$ and

$$
a \int_{x}^{\infty} u(s) d s \leq c u(x)+\int_{R} z J(z) \int_{0}^{1} u(x+t z) d t d z .
$$


It is easily seen that $J * u$ is also integrable on $(x, \infty)$. Thus (1.4) can be written as

$$
c u(x)=\int_{x}^{\infty} J * u(s) d s-\int_{x}^{\infty}(u(s)-f(u(s))) d s .
$$

To show that $v$ is integrable we argue in a similar way as before. By integrating (2.2) from $x$ to $y$ we get

$$
c \int_{x}^{y} u(s) d s \geq \int_{x}^{y}[J * v(s)-v(s)] d s+a \int_{x}^{y} v(s) d s .
$$

Since $J$ has compact support, we can again use Fubini's Theorem and Lebesgue's Theorem to obtain

$$
\int_{x}^{y} \int_{R} J(z)(v(z+s)-v(s)) d z d s \rightarrow-\int_{R} z J(z) \int_{0}^{1} v(x+t z) d t d z
$$

as $y \rightarrow \infty$. Thus $v$ is integrable and

$$
a \int_{x}^{\infty} v(s) d s \leq c v(x)+\int_{R} z J(z) \int_{0}^{1} v(x+t z) d t d z .
$$

Let $Q(x)=\int_{-\infty}^{x} J(s) d s$. Note that

$$
\int_{x}^{\infty} J * u=\int_{R} u(t) \int_{x}^{\infty} J(s-t) d s d t=\int_{R} u(t) Q(t-x) d t=\int_{R} u(s+x) Q(s) d s .
$$

For small $\delta>0$ and large $x$ for which $u(x)<\delta$ we have

$$
-\int_{x}^{\infty}(u(s)-f(u(s))) d s \geq-\left(1-f^{\prime}(0)+O(\delta)\right) \int_{x}^{\infty} u(s) d s .
$$

By integrating (2.2), we get

$$
c v(x) \geq \int_{R} v(s+x) Q(s) d s-\left(1-f^{\prime}(0)+O(\delta)\right) \int_{x}^{\infty} v(s) d s .
$$

Since $v$ is decreasing, we have the estimate

$$
\begin{aligned}
\int_{-\infty}^{0} v(s+x) Q(s) d s & \geq \int_{-\infty}^{0} v(x-s) Q(s) d s=\int_{0}^{\infty} v(s+x) Q(-s) d s \\
& =\int_{0}^{\infty} v(s+x)[1-Q(s)] d s .
\end{aligned}
$$

Thus $\int_{R} v(s+x) Q(s) d s \geq \int_{0}^{\infty} v(s+x) d s$ and we get from (2.3),

$$
\frac{c}{f^{\prime}(0)-O(\delta)} v(x) \geq \int_{x}^{\infty} v(s) d s \geq \int_{x}^{x+r} v(s) d s \geq r v(x+r) .
$$

Thus for all large $r$ there is some $k<1$ such that $v(x+r) \leq k v(x)$. Let $h(x) \equiv$ $v(x) e^{\gamma x}$, where $\gamma=\frac{1}{r} \ln \frac{1}{k}$. Then $h(x+r)=v(x+r) e^{\gamma x} e^{\gamma r} \leq v(x) e^{\gamma x}=h(x)$, and thus $h$ is bounded. This implies that $v(x)=O\left(e^{-\gamma x}\right)$ as $x \rightarrow \infty$.

Recall that $\int_{x}^{\infty} J * u(s) d s=\int_{R} J(s) v(x+s) d s$. Since $J$ has compact support and $v(x)=O\left(e^{-\gamma x}\right)$ as $x \rightarrow \infty$, also $\int_{x}^{\infty} J * u(s) d s=O\left(e^{-\gamma x}\right)$ as $x \rightarrow \infty$. Thus from (2.2) we conclude that $u(x)=O\left(e^{-\gamma x}\right)$ as $x \rightarrow \infty$.

Using Lemma 2.2 for $\lambda$ 's such that $-\gamma<\operatorname{Re} \lambda<0$ we can now define the two-sided Laplace transform of $u$ :

$$
U(\lambda) \equiv \int_{R} e^{-\lambda x} u(x) d x .
$$


Note that

$$
\int_{R} e^{-\lambda x} J * u(x) d x=\int_{R} J(y) e^{\lambda y} \int_{R} u(x+y) e^{-\lambda(x+y)} d x d y=U(\lambda) \int_{R} J(y) e^{\lambda y} d y .
$$

Since (1.4) can be written as $c u^{\prime}+J * u+\left(f^{\prime}(0)-1\right) u=f^{\prime}(0) u-f(u)$, we get

$$
(c \lambda+m(\lambda)) U(\lambda)=\int_{R} e^{-\lambda x}\left[f^{\prime}(0) u(x)-f(u(x))\right] d x,
$$

where $m(\lambda) \equiv \int_{R} e^{\lambda y} J(y) d y+f^{\prime}(0)-1$. Since $u(\infty)=0, f^{\prime}(0) u-f(u)=O\left(u^{2}\right)$ for large $x$. Hence in (2.4) the right side is defined for $\lambda$ 's such that $-2 \gamma<\operatorname{Re} \lambda<0$. We now use a property of Laplace transforms (p. 58, [13]). Since $u>0$, there exists a real $B$ such that $U(\lambda)$ is analytic for $B<\operatorname{Re} \lambda<0$ and $U(\lambda)$ has a singularity at $\lambda=B$. Hence for $c \geq c_{0}, U(\lambda)$ is defined for $\operatorname{Re} \lambda>-\alpha(c)$.

Using (2.4), we first conclude that for $0<c<c_{0}$ there are no solutions of (1.4) bounded between 0 and 1 . We argue by contradiction. Since $c \lambda+m(\lambda)$ has no real zeroes, $U(\lambda)$ is defined for all $\lambda$ such that $\operatorname{Re} \lambda<0$. Also, (2.4) can be written as

$$
\int_{R} e^{-\lambda x}\left[(c \lambda+m(\lambda)) u+f(u)-f^{\prime}(0) u\right] d x=0 .
$$

Since $c \lambda+m(\lambda) \rightarrow \infty$ as $\lambda \rightarrow-\infty$, we reach a contradiction. From now on we study the case $c \geq c_{0}$.

We recall a version of Ikehara's Theorem.

Proposition 2.3. Let $F(\lambda)=\int_{0}^{\infty} u(x) e^{-\lambda x} d x$, with u being a positive decreasing function. Assume that $F$ has the representation

$$
F(\lambda)=\frac{H(\lambda)}{(\lambda+\alpha)^{k+1}},
$$

where $k>-1$ and $H$ is analytic in the strip $-\alpha \leq \operatorname{Re} \lambda<0$. Then

$$
\lim _{x \rightarrow \infty} \frac{u(x)}{x^{k} e^{-\alpha x}}=\frac{H(-\alpha)}{\Gamma(\alpha+1)} .
$$

Proof. This version can be obtained by a modification of the proof of Theorem 2.12 in [7] (also, see pp. 54-65 for other related results). The case $k=0$ is also discussed in [13, p. 233 and [6].

We can rewrite 2.4 as

$$
\int_{0}^{\infty} u(x) e^{-\lambda x} d x=\frac{\int_{R} e^{-\lambda x}\left[f^{\prime}(0) u-f(u)\right] d x}{c \lambda+m(\lambda)}-\int_{-\infty}^{0} u(x) e^{-\lambda x} d x .
$$

Note that $\int_{-\infty}^{0} u(x) e^{-\lambda x} d x$ is analytic for $\operatorname{Re} \lambda<0$. Also, the equation $c \lambda+m(\lambda)=$ 0 does not have any zeroes with $\operatorname{Re} \lambda=-\alpha$ other than $\lambda=-\alpha$. Indeed, let $\lambda=-\alpha+i \beta$. Then $c \lambda+m(\lambda)=0$ can be written as

$$
\int_{R} e^{\alpha y} J(y) \sin ^{2} \frac{\beta y}{2} d y=0=c \beta+\int_{R} e^{\alpha y} J(y) \sin \beta y d y,
$$

a system which has a solution only for $\beta=0$.

First, let us assume that $u$ is monotone for large $x$. For $f^{\prime}(0)-1>0$, this is easily seen from (1.4). We can choose a translate of $u$ that is monotone for $x>0$. Proposition 2.3 then implies (2.1) for a suitable translate of $u$. If we do not know a priori that $u$ is monotone for large $x$, then from (1.4) we see that $c u^{\prime}-u \leq-J * u$; thus $\left(u(x) e^{-x / c}\right)^{\prime} \leq 0$ and $u(x) e^{-x / c}$ is monotone. Let $p=c^{-1}$, 
$\bar{u}(x) \equiv e^{-p x} u(x)$ and $\bar{U}(\lambda)=\int_{R} e^{-\lambda x} \bar{u}(x) d x$. Note that $\bar{U}(\lambda)=U(p+\lambda)$. We can then apply Proposition 2.3 to $\bar{u}$ to get the existence of $\lim _{x \rightarrow \infty} \frac{\bar{u}(x)}{e^{-(p+\alpha) x}}$ for $c>c_{0}$ and $\lim _{x \rightarrow \infty} \frac{\bar{u}(x)}{x e^{-(p+\alpha) x}}$ for $c=c_{0}$.

Let $u_{1}$ and $u_{2}$ denote two solutions of (1.4) bounded between 0 and 1 . Using the results in (2.1) we now construct an appropriate contraction. For $\epsilon>0$ define

$$
w_{\epsilon}(x) \equiv \frac{u_{1}(x)-u_{2}(x)}{(\epsilon|x|+1) e^{-\alpha\left(c_{0}\right) x}} \text { and } w(x) \equiv \frac{u_{1}(x)-u_{2}(x)}{e^{-\alpha(c) x}} \text { for } c>c_{0} .
$$

Note that from (2.1), $w_{\epsilon}( \pm \infty)=0$ and $w( \pm \infty)=0$. From now on we focus on the critical case $c=c_{0}$. The argument for $c>c_{0}$ follows the same lines. If $w_{\epsilon}(x) \not \equiv 0$, then, without loss of generality, we can assume there exists some finite $x_{M}^{\epsilon}$ such that $w_{\epsilon}\left(x_{M}^{\epsilon}\right)=\max _{x \in R} w_{\epsilon}(x)>0$.

Let us first assume that $x_{M}^{\epsilon} \rightarrow \infty$ as $\epsilon \rightarrow 0$. Choose $\epsilon>0$ small enough such that $x_{M}^{\epsilon}>\sup \{x: x \in \operatorname{supp} J\}$. For convenience, we simplify the notation to $x_{0} \equiv x_{M}^{\epsilon}$ and $\alpha \equiv \alpha\left(c_{0}\right)$. Note that

$$
\begin{aligned}
& u_{1}^{\prime}\left(x_{0}\right)-u_{2}^{\prime}\left(x_{0}\right) \\
& \quad=w_{\epsilon}^{\prime}\left(x_{0}\right)\left(\epsilon x_{0}+1\right) e^{-\alpha x_{0}}+w_{\epsilon}\left(x_{0}\right) \epsilon e^{-\alpha x_{0}}-w_{\epsilon}\left(x_{0}\right)\left(\epsilon x_{0}+1\right) \alpha e^{-\alpha x_{0}} .
\end{aligned}
$$

Thus, using (1.4), $w_{\epsilon}^{\prime}\left(x_{0}\right)=0$ and $\left|f\left(u_{1}\right)-f\left(u_{2}\right)\right| \leq f^{\prime}(0)\left|u_{1}-u_{2}\right|$ we get

$$
\begin{gathered}
-c_{0} w_{\epsilon}\left(x_{0}\right) \epsilon e^{-\alpha x_{0}}+c_{0} w_{\epsilon}\left(x_{0}\right)\left(\epsilon x_{0}+1\right) \alpha e^{-\alpha x_{0}} \\
\leq \int_{R} J(y) w_{\epsilon}\left(x_{0}+y\right)\left(\epsilon\left|x_{0}+y\right|+1\right) e^{-\alpha x_{0}} e^{-\alpha y} d y-\left(u_{1}-u_{2}\right)+f^{\prime}(0)\left|u_{1}-u_{2}\right| .
\end{gathered}
$$

Recall that $c_{0} \alpha=\int_{R} J(y) e^{\alpha y} d y+f^{\prime}(0)-1$ and $c_{0}=\int_{R} y J(y) e^{\alpha y} d y$. Since $u_{1}\left(x_{0}\right)-$ $u_{2}\left(x_{0}\right)>0$, after dividing by $e^{-\alpha x_{0}}$ we get

$$
\begin{gathered}
\int_{R} J(y) e^{\alpha y}\left[w_{\epsilon}\left(x_{0}\right)\left(\epsilon x_{0}-y\right)-w_{\epsilon}\left(x_{0}-y\right) \epsilon\left|x_{0}-y\right|\right] d y \\
+\int_{R} J(y) e^{\alpha y}\left[w_{\epsilon}\left(x_{0}\right)-w_{\epsilon}\left(x_{0}-y\right)\right] d y \leq 0,
\end{gathered}
$$

or, recalling that $x_{0}>\sup \{x: x \in \operatorname{supp} J\}$,

$$
\int_{R} J(y) e^{\alpha y}\left[\epsilon\left(x_{0}-y\right)+1\right]\left[w_{\epsilon}\left(x_{0}\right)-w_{\epsilon}\left(x_{0}-y\right)\right] d y \leq 0 .
$$

Let $\left[k_{1}, k_{2}\right] \subset \operatorname{supp} J$, with $k_{2}>0$. Then $w_{\epsilon}\left(x_{0}\right)=w_{\epsilon}\left(x_{0}-y\right)$ for $y \in\left[k_{1}, k_{2}\right]$. Thus, we can keep redefining $x_{0}$ until $x_{0} \in \operatorname{supp} J$, a contradiction.

If we assume that $x_{M}^{\epsilon} \rightarrow-\infty$ as $\epsilon \rightarrow 0$, then $w_{\epsilon}\left(x_{M}^{\epsilon}\right) \rightarrow 0$ as $\epsilon \rightarrow 0$. Let $w$ be as in (2.5), but with $c=c_{0}$. Since $w_{\epsilon}(x) \rightarrow w(x)$ as $\epsilon \rightarrow 0, w(x) \leq 0$ for all $x \in R$, which gives a contradiction, since $w_{\epsilon}(x) \leq w(x)$.

If we assume $\left\{x_{M}^{\epsilon}\right\}$ is bounded, then we can take a subsequence $x_{M}^{\epsilon} \rightarrow x_{1}$ as $\epsilon \rightarrow 0$, for some finite $x_{1}$. Again let $w$ be as in (2.5), but with $c=c_{0}$. From uniform convergence of $w_{\epsilon}$ to $w$ on compact sets, $w_{\epsilon}\left(x_{M}^{\epsilon}\right) \rightarrow w\left(x_{1}\right)$ as $\epsilon \rightarrow 0$. Thus $w(x) \leq w\left(x_{1}\right)$ for all $x \in R$, and we can repeat the above argument for $w(x)$ to get

$$
\int_{R} J(y) e^{\alpha y}\left[w\left(x_{1}\right)-w\left(x_{1}-y\right)\right] d y \leq 0 .
$$

Again by bootstrapping, $w\left(x_{1}\right) \equiv w\left(x_{1}-y\right)$ for all $y \in R$, and since $w(-\infty)=0$ we get $u_{1} \equiv u_{2}$. 


\section{REFERENCES}

[1] C. Atkinson and G. E. H. Reuter, Deterministic epidemic waves, Math. Proc. Cambridge Philos. Soc. 80 (1976), 315-330. MR 54:4715

[2] P. W. Bates and A. Chmaj, On a discrete convolution model for phase transitions, Arch. Rational Mech. Anal. 150 (1999), 281-305. MR 2001c:82026

[3] M. Bramson, Convergence of solutions of the Kolmogorov equation to traveling waves, Memoirs Amer. Math. Soc. 44 (1983). MR 84m:60098

[4] K. J. Brown and J. Carr, Deterministic epidemic waves of critical velocity, Math. Proc. Cambridge Philos. Soc. 81 (1977), 431-433. MR 55:10064

[5] Xinfu Chen and J.-S. Guo, Uniqueness and existence of traveling waves for discrete quasilinear monostable dynamics, Mathematische Annalen 326 (2003), 123-146. MR 2004b:37175

[6] O. Diekmann and H. G. Kaper, On the bounded solutions of a nonlinear convolution equation, Nonlinear Anal. 2 (1978), 721-737. MR 80c:45015

[7] William Ellison and Fern Ellison, Prime Numbers, A Wiley-Interscience Publication, John Wiley \& Sons, New York; Hermann, Paris, 1985. MR 87a:11082

[8] P. C. Fife, Mathematical aspects of reacting and diffusing systems, Lecture Notes in Biomathematics 28, Springer-Verlag, 1979. MR 80g:35001

[9] R. A. Fisher, The advance of advantageous genes, Ann. Eugenics 7 (1937), 355-369.

[10] A. N. Kolmogorov, I. G. Petrovsky and N. S. Piskunov, Étude de l'équation de la diffusion avec croissance de la quantité de matière et son application à un problème biologique, Bull. Univ. Moskov. Ser. Internat., Sect. A 1 (1937), 1-25. A translation in Dynamics of Curved Fronts (Perspectives in Physics Series), by Pierre Pelcé (Editor) and A. Libchaber, Academic Press, Boston, 1988. MR 91b:76002

[11] K. Schumacher, Traveling-front solutions for integro-differential equations. I, J. Reine Angew. Math. 316 (1980), 54-70. MR 81k:45007

[12] H. F. Weinberger, Asymptotic behavior of a model in population genetics, Nonlinear partial differential equations and applications (Proc. Special Sem., Indiana Univ., Bloomington, Ind., 1976-1977), 47-96. Lecture Notes in Math., Vol. 648, Springer, Berlin, 1978. MR 58:9426

[13] D. V. Widder, The Laplace Transform, Princeton University Press, Princeton, NJ, 1941. MR 3:232d

[14] J. Wu and X. Zou, Traveling wave fronts of reaction-diffusion systems with delay, J. Dynam. Differential Equations 13 (2001), 651-687. MR 2003a:35114

[15] B. Zinner, G. Harris and W. Hudson, Traveling wavefronts for the discrete Fisher's equation, J. Differential Equations 105 (1993), 46-62. MR 94k:39034

Department of Mathematics, Heriot-Watt University, Riccarton, Edinburgh, EH14 $4 \mathrm{AS}, \mathrm{UK}$

E-mail address: j.carr@ma.hw.ac.uk

Department of Mathematics, Heriot-Watt University, Riccarton, Edinburgh, EH14 $4 \mathrm{AS}, \mathrm{UK}$

Current address: Department of Mathematics, Michigan State University, East Lansing, Michigan 48824

E-mail address: chmaj@math.msu.edu 